\title{
Microbial volatile organic compounds as indicators of fungi. Can an electronic nose detect fungi in indoor environments?
}

Martyna Kuske, Anne-Claude Romain, Jacques Nicolas

Département des Sciences et Gestion de l'Environnement, Université de Liège, Avenue de Longwy 185, B-6700 Arlon, Belgium

\begin{abstract}
The paper presents a review of several studies on the detection of microbial volatile organic compounds (MVOCs) considered as indicators of fungal contamination. As fungi produce specific profiles, or fingerprints of volatile compounds, the electronic nose technology is a very promising opportunity for rapid and non costly detection of fungi in buildings. E-noses are able to distinguish between mouldy and non-mouldy samples, and also to recognise certain species of fungi. However, two limiting factors may appear decisive for employment of electronic noses in indoor fungi detection: low concentrations of MVOCs and presence of interfering substances in indoor environments.
\end{abstract}

Keywords: Indoor environment; Fungi; Detection; MVOC; Electronic nose; Sample pretreatment

\section{Introduction}

Moisture damage and subsequent microbial development are one of today's most frequent causes of health complaints in buildings. Most of the symptoms caused by moulds are not specific, and they can be easily confused with symptoms provoked by other indoor pollutants. Allergies, irritations, infections, toxic effects and general symptoms are the complaints frequently computed to fungal exposure. A relationship between the dampness and/or fungal contamination and those symptoms has been documented in many studies [1-8].

Visual inspection may be sufficient in some cases; a visible massive development of fungi, for example, yields for mitigation action regardless the results of analyses or the presence/absence of symptoms. Hidden sources of fungi are much more problematic. In such cases, the building inspection provides less pertinent information. In fact, building characteristics seem to be rarely correlated with effective humidity problems, fungal development, and/or health problems $[9,10]$.

Fungal contamination in a building has traditionally been described as quantity of viable fungi determined from air, settled dust, surface and building material samples. This method presents however several inconveniences, like long time of analyse and possibility to detect only viable airborne spores.

Therefore other possibilities to evaluate fungal contamination in indoor environment are searched. These possibilities include determination of levels of fungal components and products such as ergosterol, (1-3)-betaglucan, mycotoxins and microbial volatile organic compounds (MVOCs).

\section{Microbial volatile organic compounds}

Recently, some trends to use MVOCs analyses in investigations of damp buildings have arisen.

Moulds are reported to produce a wide range of volatile organic compounds (VOCs). Alcohols, ketones, terpenes, esters and sulphur compounds are the prevailing ones [11-13]. The volatile production is highly species dependent [12-17]; traditionally it was used to classify fungi to species level.

Some differences were also found among strains of the same species. In the study of Jelen et al. [18], the pattern of volatile sesquiterpenes was characteristic and distinctive for both toxic and non-toxic strains. Nontoxic strains of Fusarium sambucinum produced much less sesquiterpenes with less chemical diversity in comparison with the strains producing the trichothe-cenes toxins. The production of volatile terpenes seems to be linked to the formation of trichothecenes in the F. sporotrichoides cultures, and the Penicillium verrucosum strain that is capable of synthesising ochratoxin shows an accelerated production of volatile ketones compared with the nontoxigenic strain [19].

The substratum on which the fungi grow, and the environmental conditions were reported in many studies to influence the volatile production [12, 13, 15, 20-24]. Many of the volatiles are produced by only one species, and 
often on only one medium [17]. In the study of Fiedler et al. [22], more than 150 volatile substances derived from cultures of 12 fungal species have been detected. Each species had a defined MVOC profile, and the cultivation on different substrata changed the number and concentration of volatiles. Borjesson et al. [21] showed that alcohol production by Penicillium aurantiogriseum was highest during growth on oat grain, whereas the production of terpenes was most pronounced on agar substrates. This observation is concordant with the report of Rose et al. [23] who analysed VOCs from P. viridicatum and Methylobacterium mesophilicum growing on laboratory media and on component materials of automobile air conditioners. In comparison with laboratory media, fewer volatiles were detected from foam insulation materials. On laboratory media, P.viridicatum produced mainly terpenes and alcohols; on insulation materials, it produced offensive smelling alcohols and esters, such as 2-methyl-1-propanol and 3-penten-2-ol.

The highest MVOC production associated with secondary fungal metabolism (specifically terpenes and sesquiterpenes) seems to occur prior to and during sporulation and mycotoxin production [25]. In general, conditions favouring growth also favour the production of secondary volatile metabolites [26], but factors that favour the production of one VOC may be unfavourable to the production of another one [27]. Various VOCs are products of microbial activity, however no single VOC seems to be a reliable indicator of biocontamination in building materials [28]. Some of the volatile compounds are detected more often than others, and these compounds could eventually indicate fungal contamination in buildings. In fact, VOC analysis by GC/SM in a building with a history of Sick Building Syndrome symptoms and microbial growth revealed unusual levels of several MVOCs: 2-methyl-1-propanol, dimethyl disulfide, dimethyl trisulfide, dimethyl tetrasulfide and 2ethylhexanol [29]. The authors found that using MVOCs as mould growth indicators necessitates some kind of control or "normal" levels for the selected volatile compounds. This is difficult because few MVOCs are unique for microbial growth, and some of them (geosmin and 2-methylisoborneol, for example) are often not detected.

From several so-called MVOCs analysed by Elke et al. [30] in damp and mouldy houses, 3-methylbutan-1-ol, hexan-2-one, heptan-2-one and octan-3-ol were found to be the most reliable indicators for mould formation. A correlation was found between Aspergillus sp, and heptan-2-one, hexan-2-one, octan-3-ol, octan-3-one and alpha-terpineol, while the occurrence of Eurotium $s p$. was correlated with higher indoor air concentrations of 3methylbutan-1-ol, 3-methylbutan-2-ol, heptan-2-one, hexan-2-one, octan-3-ol and thujopsene.

Ström et al. [31] investigated VOCs as a means to detect the occurrence of fungi and bacteria within building constructions. Fifteen compounds considered to be selectively produced by fungi and bacteria were measured in houses suffering from indoor complaints, in reference houses, and outdoors: 3-methylfuran, 3-methyl-1-butanol, 3-methyl-2-butanol, 2-methyl-1-propanol, 2-pentanol, 2-hexanone, 2-heptanone, 3-octanone, 1-butanol, 3octanol, 1-octen-3-ol, 2-octen-1-ol, 2-methy-lisoborneol, geosmin and 2-isopropyl-3-methoxypyra-zine. A significant increase was observed in the concentration of MVOC in houses with microbial odour problems compared with unaffected houses and outdoor air samples.

Some recent studies have shown that commonly used MVOCs indicate the presence of excess moisture in building materials rather than exclusive fungal growth [32]. However, considering the high frequency of microbial growth in humid materials, MVOCs can be used in detection of hidden biocontamination as well as in detection of moisture problems, risk of fungal development and odour complaints in buildings.

The great diversity of microbial volatile production, that has been reported in the literature, make the selection of fungal indicators quasi-impossible. The selection becomes especially difficult by the fact that no compound was found to be produced by every species in any conditions. The detection of moulds cannot be thus based on the presence of a single substance but on the coexistence of several relevant compounds. Actually, it has been suggested that MVOC diagnosis should be based on the presence of several compounds forming characteristic patterns [11,22,32-34]. These compounds, most often reported in different studies, are alcohols, ketones, terpenes, furanes, and sulphur compounds (Table 1).

In all mentioned studies, the detection of MVOCs was carried out by sampling into carbon-based or TENAX adsorbents, and analysing by gas chromatography and mass spectrometry. This method, very specific and sensitive, requires however experience and special laboratory equipment, takes time and is relatively expensive. In looking for rapid and simple methods to detect microbial contamination, researchers got interested in an instrument called "electronic nose". Regarding the working principle of electronic nose, this instrument should be able to recognise the patterns of compounds related to the presence of fungi or excessive moisture. 
Table 1: Microbial volatile organic compounds frequently reported in the literature

\begin{tabular}{lll}
\hline Chemical group & Compound & Reference \\
\hline Alcohols & 2-methyl- 1-propanol & {$[11,35,12,16,36,39,31,17,13,42]$} \\
& 3-methyl- 1-butanol & {$[11,12,28,16,36,39,31,17,13,40-42]$} \\
& 2-methyl- 1-butanol & {$[11,12,17]$} \\
& 3-octanol & {$[12,37,31,17]$} \\
& 1-octen-3-ol & {$[11,35,12,28,36,19,39,31,17,40,41]$} \\
& 1-hexanol & {$[28,16,13]$} \\
& 1-pentanol & {$[28,36]$} \\
& 2-methyl-isoborneol & {$[19,31]$} \\
& Geosmin & {$[35,16,39,31,17]$} \\
Terpenes and & Limonene & {$[11,28,19]$} \\
Sesquiterpenes & Pinene & {$[11,16,19]$} \\
Ketones & & {$[17,42]$} \\
& 3-octanone & {$[28,16,36,38,39,31,17,40]$} \\
Furanes & 2-heptanone & {$[35,16,31,13,42]$} \\
Sulphur compounds & Dimethyl disulfide & {$[35,17]$} \\
& &
\end{tabular}

\section{Electronic nose}

An electronic nose tries to mimic the three phases of the human olfactory system: detection, signal processing and recognition/interpretation of odours. The sensors employed in electronic noses are non-specific. It means that they are not selective to a given chemical compound, but sensible to nearly all compounds, and slightly more to one chemical family, such as organic solvents, fatty acids, sulfurous gases, etc. This way, the responses of the sensors produce patterns characteristic for each chemical mixture presented to the sensor array. By presenting many different chemicals to the sensor array, a database of patterns or signatures is built up. This database is used to train the pattern recognition system, and afterwards it allows recognising one from the odours stored in the memory.

As an odour is a mixture of gaseous compounds at relatively low concentrations, the electronic nose principle can be applied in detection and monitoring of any gaseous mixture, even if this mixture does not smell.

Electronic noses are already used in several domains, mainly in food industry. They found application in discriminating between different kinds of coffee, in detecting spoiled fish, in classifying cheese, vegetable oil or beer, etc. In the past few years, some studies showed utility of electronic nose to detect diseases of metabolic or microbial origin. Ping et al. [43] analysed the breath of 18 diabetic and 14 normal volunteers before and after meal. The results showed that analysis of expired air after the test meal might be a reliable monitoring method. Odours over the headspaces of leg ulcers have been analysed with an electronic nose comprising 20 conductive polymers [44]. A two-dimensional odour map demonstrated clear differences between ulcers infected with Staphylococcus aureus and with Streptococcus species. In 1997, Hanson and Steinberg [45] reported the use of a 32-sensor array electronic nose to diagnose the presence of pulmonary infections. He obtained a good discrimination between nine infected and ten uninfected intensive care patients.

These and several other studies [46-51] prove that an electronic nose reacts on volatile products of microbial metabolism, and that it can detect the presence of microorganisms in different kinds of samples.

\section{Electronic nose for fungal detection}

Electronic noses seem to be particularly useful in cereal grains qualification. The information on absence or presence of fungi and mycotoxins is required immediately, and commonly used biological methods give the results only after few days. Therefore the possibilities to use electronic nose for fungal detection were examined mainly in this domain. The studies show that sensor arrays and different techniques of statistical analysis should 
allow detecting and classifying selected fungal species.

Jonsson et al. [48] used an electronic nose, consisting of different types of sensors, to analyse samples of oats, rye, barley and wheat with different odours, different levels of ergosterol and colony forming units (CFUs). A pattern recognition technique based on an artificial neural network (ANN) could predict the odour classes of good, mouldy, weakly and strongly musty oats with a high degree of accuracy. In wheat, ANN predictions were correlated with measured ergosterol and with fungal and bacterial CFUs.

Olsson et al. [38] examined the possibility of using electronic nose or gas chromatography combined with mass spectrometry (GC-MS) to quantify ergosterol and CFUs of naturally contaminated barley samples. Three of 40 samples were misclassified using data from the electronic nose, while data from analysis of the volatile compounds detected with the GC-MS led to six misclassified samples. Regression models (partial least-squares (PLS)) were built to predict ergosterol- and CFU-levels with data from the GC-MS and electronic nose measurements. PLS models based on both GC-MS and electronic nose data could be used to predict the ergosterol levels with high accuracy and with low mean square error of prediction. CFU values could not be predicted with the same degree of confidence.

In 2002, Olsson et al. [52] compared the presence of mycotoxins with the presence of VOCs in grain samples. The samples were evaluated with regard to moisture content, fungal contamination, ergosterol content and levels of two mycotoxins: ochratoxin A and deoxynivalenol. Volatile compounds were analysed using gas chromatography and an electronic nose. Data were evaluated by multivariate data analyses such as principal component analysis (PCA) and PLS. Using the volatile compounds detected and quantified by either GC-MS or the electronic nose, it was possible to classify the samples according to the content of mycotoxins below or above the maximum limit established by the Swedish National Food Administration (for ochratoxin A: $5 \mu \mathrm{g} / \mathrm{kg}$ cereal grain). The electronic nose misclassified seven, whereas the GC-MS system misclassified three of 37 samples.

Six spoilage fungi (four Eurotium sp., a Penicillium sp. and a Wallemia sp.) isolated from cereal grain were cultivated on laboratory medium and analysed with an electronic nose consisting of 14 polymer sensors [53]. Normalized data were analysed by PCA, discriminant function analysis (DFA) and cluster analysis. Five of the species could be differentiated using this approach, while the related species of Eurotium amstelodami and $E$. herbariorum could not, at an early stage of growth $(48 \mathrm{~h})$. Better detection patterns were observed after $72 \mathrm{~h}$ incubation. With the same electronic nose system, Keshri et al. [54] were able to differentiate between mycotoxigenic and non-mycotoxigenic strains of Fusarium moniliforme and F. proliferatum, and thus to answer whether the grain is not dangerous for consumption.

\section{Electronic nose for indoor mould detection}

The ability of electronic noses to distinguish between good and mouldy cereals permits thinking that they can find application in indoor fungal detection. The idea is quite new, and has not been well examined so far. Some recent studies prove however that this new application is feasible.

Schiffman et al. [55] investigated the possibility to detect and classify five fungal species commonly found in indoor environments, using an electronic nose. An array of 15 metal oxide sensors was used for measuring odour in air samples, and the data were analysed with specially developed signal processing algorithms. Linear discriminant analysis (LDA), $k$ nearest neighbours $(\mathrm{KNN})$ and least square analysis (LS) were employed to classify the data. The electronic nose was capable of discriminating among the fungi with up to $96 \%$ accuracy. It was also able to recognise selected five VOCs that are emitted by fungi (ethanol, 2-methyl-1-propanol, 3pentanone, 3-octanol and 3-octanone).

Persaud et al. [56] used an array of 32 sensors for detection of dry rot wood decay fungi. The system utilises solid phase microextraction headspace sampling in direct combination with metal oxide semiconductor sensor technology. Mass spectrometry detection results for pine in the early stages of infection have identified volatiles that are mostly terpenoid in nature, the most predominant of which are: carene, fenchone, camphor, limonene and 3-isopropyl toluene [57]. Laboratory trials have indicated that sensor sensitivity to VOC emission accompanying Serpula lacrimans infection of pine timber was sufficient to enable discrimination between infected and uninfected samples (Fig. 1).

Schreiber [58] investigated the possibility to evaluate perceived indoor air quality and to detect the presence of fungi in buildings with electronic noses. He used two commercial e-noses-MOSES II and KAMINA. The 
results of the experiments with MOSES II showed that an assessment of the perceived air quality was possible with the e-nose. In the experiment with three mould species: Aspergillus niger, A. versicolor and Cladosporium sphaerospermum grown on malt-extract agar, the PCA permitted to determinate that a mould is present, but it did not permit to differentiate among different species. The author suggested that the results should be improved by indirect sampling and another more reproducible experiment set-up.

Fig. 1: Principal component analysis of normalised sensor responses for Serpula lacrymans infected and uninfected samples (from Persaud et al. [56]).

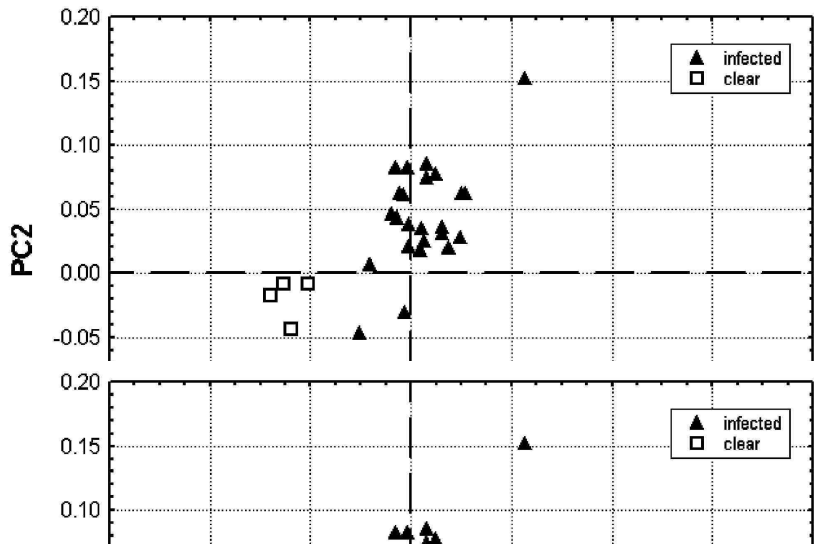

\section{Discussion and trends}

VOCs seem to be interesting indicators of mould presence in buildings. In comparison with biological methods, a classical analyse of MVOCs by GC-MS is faster since the results can be obtained the same day. Using an electronic nose could even shorten this time to several minutes. MVOCs analyse permits to detect fungi in their early stage of development, before the production of spores. Furthermore, it allows detecting moulds that do not produce much spores or don't liberate them into the air, as well as moulds growing in poorly accessible and invisible areas, for example behind a wall paper. In fact, barriers such as vinyl wallpaper and polyethylene sheeting may impede spore transport whereas they do not prevent the diffusion of MVOCs [31]. In such cases, biological methods will give negative results, and the presence of fungi can be detected only with other methods.

One of the difficulties to use MVOCs as mould indicators is the great variety of substances produced by fungi. The choice of the compounds is a difficult task and should be based rather on a complex of substances forming a kind of "fingerprints" characteristic for moulds, than on individual compounds. Electronic nose technology responds well to this requirement since it utilises the differences of compounds' profiles to distinguish among different chemical mixtures.

Low concentrations found generally indoors can be a limiting factor necessitating a preconcentration of the sample. Ström et al. [31] measured, using gas chromatography-mass spectrometry, concentrations of MVOCs in houses with microbial odour problems, in reference houses and outdoors. In contaminated buildings, the total concentration of 15 VOCs considered to be selectively produced by micro-organisms varied between 50 and $100 \mu \mathrm{g} / \mathrm{m}^{3}$. In reference houses, the total concentration of the same compounds was only slightly higher than in outdoor samples, and was about $10 \mu \mathrm{g} / \mathrm{m}^{3}$. The laboratory of Air Quality Sciences in the USA reported, on base of 600 samples from different buildings, that the average total MVOC level was $27 \mu \mathrm{g} / \mathrm{m}^{3}$ with a maximum of $2000 \mu \mathrm{g} / \mathrm{m}^{3}$ [59].

The detection limit of most sensors exceeds several or several tens of $\mu \mathrm{g} / \mathrm{m}^{3}$. If a single sensor was sensible on all MVOCs, their total concentration should be enough high to provoke the sensor's reaction. However, as the sensors show some selectivity, they will react on only some of the compounds, and the concentrations of the microbial compounds may appear too low to be detected. In order to increase the sensitivity of the electronic nose, a system of sample pretreatment can be necessary.

Two different sample pretreatment methods have been examined with e-noses: preconcentration, and separation of compounds. Sample preconcentration should bring as a result the concentrations of compounds enough high to provoke sensors' reaction. Separation, on the other hand, should allow the compounds of interest to pass through while preventing interfering substances to reach the sensors. 
Membrane separators are often employed in analytical instruments, where they can function as separators and purifiers. Nafion membrane separator is an example of such a system. Nafion is a fluoropolymer with ion exchange capacity, and removes not only water but also volatile alcohols and some other polar solvents. Stetter et al. [60] used a Nafion drier to remove water vapour from samples. Using the Nation membrane improved sensitivity of the electronic nose (MOSES II) to detect and discriminate coliform bacteria [60,61]. The improvement in the analytical capability was obtained here by suppressing the background that was common to all samples. The components removed by Nation, particularly alcohols, are one of the most important substances that could allow differentiating between healthy and mould infected buildings. Thus, the Nation drier will not be a suitable sample preparation in the case of fungal detection in indoor environments.

A great number of analytical methods use preconcentration techniques. The compounds are adsorbed on an adsorbent, and then chemically or thermally desorbed. Thermal desorption systems are commonly used for analyses of trace air contaminants by gas chromatography.

Such systems are however too big for portable electronic nose applications. Strathmann [62] have designed and tested several thermal desorption set-ups. The final setup was made up of a small tube containing $230 \mathrm{mg}$ of a commercially available adsorbent raisin (e.g. Tenax). Two heater elements enclosed the tube and ensured even temperature distribution along the trap. The system was capable of reaching $230^{\circ} \mathrm{C}$ within $2: 30 \mathrm{~min}$. This preconcentration set-up improved the sensitivity of the sensor system.

Glass or stainless steel capillaries packed with 1-10mg adsorbent were tested by Lu et al. [63] in preconcentrators prior to an array of polymer-coated surface acoustic wave sensors. The system permitted efficient chromatographic separation and low-parts-per-billion detection limits. It thus appear adequate for indoor air quality assessments for VOCs.

\section{Conclusion}

Recent studies prove that detection of moulds by analysing microbial volatile organic compounds (MVOCs) is possible. In laboratory experiments, electronic noses could distinguish between sterile and contaminated samples. Mould detection in indoor environments is however more complex. A great number of substances emitted by different sources inside buildings can interfere in the analysis. In fact, many substances produced by fungi are also emitted by building materials, and the quantities produced by micro-organisms may be much lower than those emitted by other sources. This fact is particularly important for the e-nose technology since the sensors' sensibility may appear too low to detect small changes in concentrations of compounds belonging to the same chemical group. Sample preconcentration should improve the results of the e-nose. Of the sampling techniques offering a noticeable concentration effect, adsorption followed by thermal desorption appear to be most suited for electronic noses. Commercial thermal desorbers presently used are not adapted for electronic noses - they are big and complicated, and need adequate laboratory equipment. Small systems able to attain the temperature of $250{ }^{\circ} \mathrm{C}$ in few seconds should be developed, and the home-made prototypes seem enough powerful.

Preconcentration of searched compounds means however higher concentrations of interfering substances as well. These interfering substances are emitted by many sources, including the support on which fungi grow. In some cases, interfering substances may appear preponderant in such a degree that the MVOCs become "hidden". Since the sensors used in e-noses are nonspecific, the total concentration of a particular chemical group will be more relevant than concentrations of every substance. Presuming that MVOCs contribute significantly in total concentration of particular chemical groups, the pattern of compounds in presence of moulds should be sufficiently distinct to be recognised by an electronic nose. This aspect has not been evaluated so far, and it should become a prior subject in the investigations of electronic noses used for mould detection.

\section{References}

[1] Brunekreff B, Verhoeff AP, van Strien RT, Wijnen, JH. Home dampness and childhood respiratory symptoms: the role of sensitization to dust mites and moulds. In: Samson, et al., editors. Health implications of fungi in indoor environments; 1994. p. 189-199.

[2] Johanning E, Biagini R, Hull D, Morey P, Jarvis B, Landsbergis P. Health and immunology study following exposure to toxigenic fungi (Stachybotrys chartarum) in a water-damaged office environment. International Archives of Occupational and Environmental Health 1996;68:207-18. 
[3] Klanova K. The concentrations of mixed populations of fungi in indoor air: rooms with and without mould problems; rooms with and without health complaints. Central European Journal of Public Health 2000;8:59-61.

[4] Koskinen O, Husman T, Hyvärinen A, Reponen T, Nevalainen A. Two mouldy day-care centers: a follow-up study of respiratory symptoms and infections. Indoor Air 1997;7:67-76.

[5] Norback D, Wieslander G, Nordstrom K, Walinder R. Asthma symptoms in relation to measured building dampness in upper concrete floor construction, and 2-ethyl-1-hexanol in indoor air. International Journal of Tuberculosis and Lung Disease 2000;4:1016-25

[6] Walinder R, Norback D, Johanson G. Pulmonary reactions after exposure to 3-methylfuran vapour, a fungal metabolite. International Journal of Tuberculosis and Lung Disease 1998;2:1037-9.

[7] Wang GH, Li CS. Indoor endotoxin and glucan in association with airway inflammation and systemic symptoms. Archives of Environmental Health 1999;54:172-9.

[8] Wilson CE. Sudden infant death syndrome and Canadian Aboriginals: bacteria and infections. FEMS Immunology and Medical Microbiology 1999;25:221-6.

[9] Wickman M, Gravesen S, Nordvall SL, Pershagen G, Sundell J. Indoor viable dust-bound microfungi in relation to residential characteristics, living habits, and symptoms in atopic and control children. Journal of Allergy and Clinical Immunology 1992;89: 2-759.

[10] Verhoeff AP, Van Wijnen JH, Van Reenen-Hoextra ES, Samson RA, Van Strien RT, Brunekreef B. Fungal propagules in house dust II. Relation with residential characteristics and respiratory symptoms. Allergy 1994;49:540-7.

[11] Fischer G, Muller T, Schwalbe R, Ostrowski R, Dott W. Exposure to airborne fungi, MVOC and mycotoxins in bio-waste-handling facilities. International Journal of Hygiene and Environmental Health 2000;203:97-104.

[12] Kiviranta H, Tuomainen A, Reiman M, Laitinen S, Liesivuori J, Nevalainen A. Qualitative identification of volatile metabolites from two fungi and three bacteria species cultivated on two media. Central European Journal of Public Health 1998;6:296-9.

[13] Sunesson AL, Nilsson CA, Andersson B, Blomquist G. Volatile metabolites produced by two fungal species cultivated on building materials. Annals of Occupational Hygiene 1996;40:397-410.

[14] Fischer G, Muller T, Moller M, Ostrowski R, Dott W. MVOC of fungi-use as an indicator for exposure level. Schriftenreihe des Vereins fur Wasser-, Boden- und Lufthygiene 1999;104:183-92.

[15] Horner W, Worthan A, Epstein B, Black M. MVOC emission patterns of indoor fungi: strain and culture differences, (online 1999) http://www.aiha.org/abs99/9pos3.html.

[16] Korpi A, Pasanen A-L, Viitanen H. Volatile metabolites of Serpula lacrymans, Coniophora puteana Poria placenta, Stachy-botrys chartarum and Chaetomium globosum. Building and Environment 1999;34:205-11.

[17] Sunesson A-L, Vaes WHJ, Nilsson CA, Blomquist G, Andersson B, Carlson R. Identification of volatile metabolites from five fungal species cultivated on two media. Applied and Environmental Microbiology 1995;61:2911-8.

[18] Jelen H, Mirocha CJ, Wasowicz E, Kaminski E. Production of volatile sesquiterpenes by Fusarium sambucinum strains with different abilities to synthesize trichothecenes. Applied and Environmental Microbiology 1995;61:3815-20.

[19] Pasanen A-L, Lappalainen S, Pasanen P. Volatile organic metabolites associated with some toxic fungi and their mycotox-ins. Analyst $1996 ; 121: 1949-53$

[20] Bjurman J, Kristensson J. Volatile production by Aspergillus versicolor as a possible cause of odor in houses affected by fungi. Mycopathologia 1992;118:173-8.

[21] Borjesson T, Stollman U, Schnurer J. Volatile metabolites and other indicators of Penicillium aurantiogriseum growth on different substrates. Applied and Environmental Microbiology 1990;56:3705-10.

[22] Fiedler K, Schutz E, Geh S. Detection of microbial volatile organic compounds (MVOCs) produced by moulds on various materials. International Journal of Hygiene and Environmental Health 2001;204:2-3.

[23] Rose LJ, Simmons RB, Crow SA, Ahearn DG. Volatile organic compounds associated with microbial growth in automobile air conditioning systems. Current Microbiology 2000;41:206-9.

[24] Gao P, Korley F, Chen B. Profiles of volatile metabolites produced by five Aspergillus species cultivated on malt extract agar and gypsum board, (online 1999) http://www.aiha.org/ abs99/9iaq2.html.

[25] Zeringue HJ, Bhatnagar D, Cleveland TE. C15H24 volatile compounds unique to aflatoxigenic strains of Aspergillus flavus. Applied and Environmental Microbiology 1993;59:2264-70. 
Published in: Building and Environment (2005), vol.40, iss.6, pp. 824-831

Status: Postprint (Author's version)

[26] Frisvad JC, Bridge PD, Arora DK. Chemical fungal taxonomy. New York: Marcel Dekker Inc.; 1998.

[27] Korpi A. Fungal volatile metabolites and biological responses to fungal exposure. Doctoral dissertation 2001, Department of Environmental Sciences, University of Kuopio; 2001

[28] Korpi A, Pasanen A-L, Pasanen P. Volatile compounds originating from mixed microbial cultures on building materials under various humidity conditions. Applied and Environmental Microbiology 1998;64:2914-9.

[29] Wilkins K, Hansen MO, Larsen K. MVOC analysis for detection of microbial growth in buildings II. A new sampling method? (online 1999) http://www.asc.amr.dk/forskning/43rapporter/ unders_bygn/Under_byg.pdf.

[30] Elke K, Begerow J, Oppermann H, Kramer U, Jermann E, Dunema L. Determination of selected microbial volatile organic compounds by diffusive sampling and dual-column capillary GC-FID-a new feasible approach for the detection of an exposure to indoor mould fungi? Journal of Environmental Monitoring 1999;5:445-52.

[31] Strom G, West J, Wessen B, Palmgren U. Quantitative analysis of microbial volatiles in damp Swedish houses. In: Flannigan, et al., editors. Health implications of fungi in indoor environments. Amsterdam: Elsevier; 1994. p. 291-305.

[32] Pasanen A-L, Korpi A, Kasanen J-P, Pasanen P. Critical aspects on the significance of microbial volatile metabolites as indoor air pollutants. Environment International 1998;24:703-12.

[33] Bjurman J, Nordstrand E, Kristensson J. Growth-phase-related production of potential volatile-organic tracer compounds by moulds on wood. Indoor Air 1997; 7:2-7.

[34] Wilkins K, Nielsen EM, Wolkoff P. Patterns in volatile organic compounds in dust from mouldy buildings. Indoor Air 1997;7: 128-34.

[35] Keller R, Senkpiel K, Ohgke H. Use of MVOC measurements and odor perception as indicator of mould in indoor areas. In: Bioaerosols, fungi and mycotoxins: health effects, assessement, prevention and control, E. New York: Johanning; 1999.

[36] Larsen TO, Frisvad J. Production of volatiles and presence of mycotoxins in conidia of common indoor Penicillia and Aspergillii. In: Flannigan, et al., editors. Health implications of fungi in indoor environments. Amsterdam: Elsevier; 1994. p. $251-279$.

[37] Martin J, Gao P. Volatile metabolites produced by Stachybotrys Chartarum on rice and gypsum board, (online 2000) http:// www. aiha. org/abs00/0iaq. html.

[38] Olsson J, Borjesson T, Lundstedt T, Schnurer J. Volatiles for mycological quality grading of barley grains: determinations using gas chromatography-mass spectrometry and electronic nose. International Journal of Food Microbiology 2000;59:167-78.

[39] Schnurer J, Olsson J, Borjesson T. Fungal volatiles as indicators of food and feeds spoilage. Fungal Genetics and Biology 1999;27:209-17.

[40] Tuma D, Sinha RN, Muir WE, Abramson D. Odor volatiles associated with microflora in damp ventilated and non-ventilated bin-stored bulk wheat. International Journal of Food Microbiology 1989;8:103-19.

[41] Wilkins CK, Scholl S. Volatile metabolites of some barley storage molds. International Journal of Food Microbiology 1989;8:11-7.

[42] Wilkins K, Larsen K. Variation of volatile organic compound patterns of mould species from damp buildings. Chemosphere $1995 ; 5: 3225-36$

[43] Ping W, Yi T, Haibao X, Farong S. A novel method for diabetes diagnosis based on electronic nose. Biosensors and Bioelectronics $1997 ; 12: 1031-6$

[44] Parry AD, Oppenheim B. Leg ulcer odour detection identifies beta-haemolytic streptococcal infection. Journal of Wound Care 1995;4:404-6.

[45] Hanson III CW, Steinberg HA. The use of a novel "electronic nose" to diagnose the presence of intrapulmonary infection. Anesthesiology 1997;87A269.

[46] Blixt Y, Borch E. Using an electronic nose for determining the spoilage of vacuum-packaged beef. International Journal of Food Microbiology 1999;46:123-34.

[47] Du WX, Kim J, Cornell JA, Huang T, Marshall MR, Wei CI. Microbiological, sensory, and electronic nose evaluation of yellowfin tuna under various storage conditions. Journal of Food Protection 2001;64:2027-36.

[48] Jonsson A, Winquist F, Schnurer J, Sundgren H, Lundstrom I. Electronic nose for microbial quality classification of grains. International Journal of Food Microbiology 1997;35:187-93.

[49] Keshri G, Voysey P, Magan N. Early detection of spoilage moulds in bread using volatile production patterns and qualitative enzyme assays. Journal of Applied Microbiology 2002;92:165-72. 
Published in: Building and Environment (2005), vol.40, iss.6, pp. 824-831

Status: Postprint (Author's version)

[50] Marsili RT. SPME-MS-MVA as an electronic nose for the study of off-flavors in milk. Journal of Agricultural and Food Chemistry 1999;47:648-54

[51] Pavlou AK, Magan N, Sharp D, Brown J, Barr H, Turner AP. An intelligent rapid odour recognition model in discrimination of Helicobacter pylori and other gastroesophageal isolates in vitro. Biosensors and Bioelectronics 2000;15:333-42.

[52] Olsson J, Borjesson T, Lundstedt T, Schnurer J. Detection and quantification of ochratoxin A and deoxynivalenol in barley grains by GC-MS and electronic nose. International Journal of Food Microbiology 2002;72:203-14.

[53] Keshri G, Magan N, Voysey P. Use of an electronic nose for the early detection and differentiation between spoilage fungi. Letters in Applied Microbiology 1998;27:261-4.

[54] Keshri G, Magan N. Detection and differentiation between mycotoxigenic and non mycotoxigenic strains of two Fusarium spp. using volatile production profiles and hydrolytic enzymes. Journal of Applied Microbiology 2000;89:825-33.

[55] Schiffman ss, Wyrick DW, Payne GA, O'Brian G, Nagle, HT. Effectiveness of an electronic nose for monitoring bacterial and fungal growth. In: Proceedings of ISOEN 2000, Brighton, July 20-24, 2000. p. 173-80.

[56] Persaud KC, Wareham PD, Payne PA, Chueh H, Hatfield JV. A portable electronic nose for dry rot detection, In: Actes des conferences "La métrologie des odeurs et les nez électroniques", Eurodeur - Airodeur 2001, Paris.

[57] Hatfield JV, Daniels AR, Snowden D, Persaud KC, Payne PA. Development of a hand held electronic nose (H2EN). In: Proceedings of the 13th European conference on solid-state transducers, The Hague, The Netherlands, 12-15 September, 1999. p. $215-8$.

[58] Schreiber FW. Perceived air quality: investigation of the non-sensory odor assessment in indoor environments. Doctoral thesis, School of Process Sciences and Engineering of the Technical University of Berlin; 2000.

[59] Air Quality Sciences. Microbial MVOCs. Airfaqs 2000; 8(2):4.

[60] Stetter JR, Strathmann S, McEntegart C, Decastro M, Penrose WR. New arrays and sampling systems for a modular electronic nose. Sensors and Actuators B 2000;69:410-9.

[61] McEntegart CM, Penrose WR, Strathmann S, Stetter JR. Detection and discrimination of coliform bacteria with gas sensor arrays. Sensors and Actuators B 2000;70:170-6.

[62] Strathmann S. Sample conditioning for multi-sensor systems. Doctoral thesis, Faculty of Chemistry and Pharmacy, Eberhard-Karls University of Tübingen; 2001, p. 176

[63] Lu C-J, Zellers ET. A dual-adsorbent preconcentrator for a portable indoor-VOC microsensor system. Analytical Chemistry 2001;73:3449-57. 